\title{
Sonderheft Beiträge zur Mechatronik
}

\author{
Hans Irschik \\ Institut für Technische Mechanik, Johannes Kepler Universität Linz, Linz, Österreich
}

Online publiziert 18. Oktober 2016

Das Wort "Mechatronik“ wurde zunächst in Japan aus der industriellen Tätigkeit heraus erfunden und vermarktet, siehe z.B. [1], wo es heißt: „The term 'mechatronics' was coined by Tetsuro Mori, the senior engineer of the Japanese company Yaskawa in 1969." Das High-Tech Unternehmen Yaskawa ließ sich diesen Begriff daraufhin mehrere Jahre lang als Markenzeichen schützen. Nach Ablauf dieses Schutzes entwickelte sich die Mechatronik unter Zusammenwirken von Industrie und Academia zu einem eigenständigen ingenieurwissenschaftlichen Fach in Lehre und Forschung. Die französische Norm definiert dieses Fach zum Beispiel wie folgt: „approach aiming at the synergistic integration of mechanics, electronics, control theory, and computer science within product design and manufacturing, in order to improve and/or optimize its functionality,, [1]. Das weltweit erste Universitäts-Diplomvollstudium für Mechatronik wurde 1990 an der Johannes Kepler Universität Linz (JKU Linz) eingerichtet, siehe zum Beispiel [2] für die Entwicklung des zugehörigen Fachbereichs bis 2010. Seit dem Jahr 2000 betreibt der JKU-Fachbereich Mechatronik gemeinsam mit der Industrie die "Linz Center of Mechatronics $\mathrm{GmbH}_{\text {, siehe }}$ [3], an welchem ein COMET-K2 Kompetenzzentrum beheimatet ist, wo vor-wettbewerbliche, geförderte und durch ausländische Fachexperten beurteilte Forschungen durchgeführt werden. Das vorliegende Sonderheft enthält aktuelle Berichte aus diesem COMET-K2 Bereich, mit besonderer Berücksichtigung von Anwendungen im Maschinenund Anlagenbau (eine Einschränkung, die aus Platzgründen notwendig war). Die Beiträge demonstrieren unter anderem, dass sich die Mechatronik in den letzten Jahren verstärkt der Anwendung neuer industrieller Konzepte, wie "Internet der Dinge”, "Industrie 4.0" und „Cyber Physical Systems", auf komplexe Maschinen und Prozesse widmet, bei welchen zum Beispiel die in diesen Konzepten geforderte computergestützte Beschreibung der physikalischen Vorgänge in Echtzeit ohne tiefer gehende Forschungen nicht möglich werden kann.

H. Irschik $(\bowtie)$

Institut für Technische Mechanik

Johannes Kepler Universität Linz,

Altenbergerstraße 69 ,

4040 Linz, Österreich

hans.irschik@jku.at

\section{Danksagung}

Die Beiträge in diesem Sonderheft wurden im Rahmen eines K2 Zentrums in der Linz Center of Mechatronics GmbH (LCM) durchgeführt. Die K2 Projekte werden aus Mitteln des österreichischen COMET-K2 Programms finanziert. Die COMET-K2 Projekte im LCM werden unterstützt von der Österreichischen Bundesregierung, dem Land Oberösterreich, der Johannes Kepler Universität und allen wissenschaftlichen Partnern, die Teil des K2-COMET Konsortiums innerhalb des LCM sind.

Interessenkonflikt. H. Irschik gibt an, dass kein Interessenkonflikt besteht.

\section{Literatur}

1. https://en.wikipedia.org/wiki/Mechatronics (03.10.2016)

2. Irschik, H.: Mechatronik - Ausbildung und technischer Fortschritt, Österreichische Ingenieur- und Architekten-Zeitschrift (ÖIAZ), 157 (2012), pp 69-75

3. http://www.Icm.at (03.10.2016)

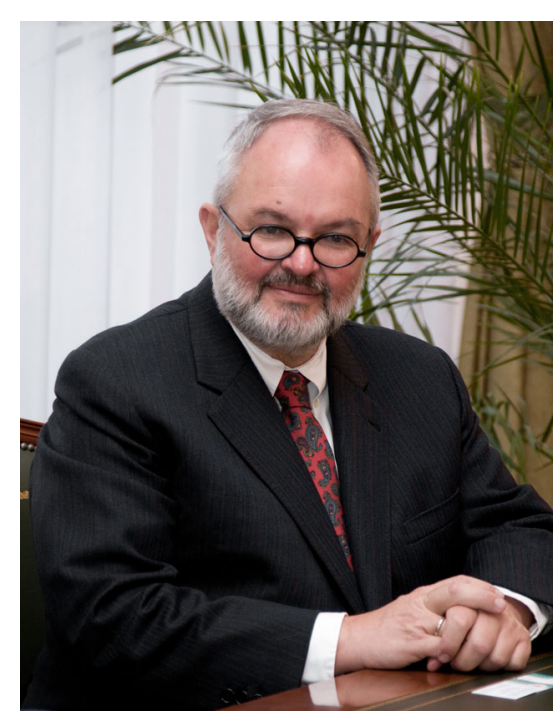

Hans Irschik 